\title{
Molecular cloning and characterization of plerocercoid-immunosuppressive factor from Spirometra erinaceieuropaei
}

\author{
Yoko Kondo ${ }^{\mathrm{a}}$, Daisuke Ito ${ }^{\mathrm{a}}$, Sayuri Tademoto ${ }^{\mathrm{b}}$, Nanase Itami ${ }^{\mathrm{a}}$, Shuma Nishikata ${ }^{\mathrm{a}}$, \\ Eizo Takashima ${ }^{c}$, Takafumi Tsuboi ${ }^{\mathrm{c}}$, Soji Fukumoto ${ }^{\mathrm{a}, \mathrm{d}}$, Hitoshi Otsuki ${ }^{\mathrm{a}, *}$ \\ ${ }^{a}$ Division of Medical Zoology, Department of Microbiology and Immunology, Faculty of Medicine, Tottori University, Yonago 683-8503, Japan \\ ${ }^{\mathrm{b}}$ Technical Department, Tottori University, Yonago 683-8503, Japan \\ ${ }^{c}$ Division of Malaria Research, Proteo-Science Center, Ehime University, Matsuyama 790-8577, Japan \\ ${ }^{\mathrm{d}}$ Tottori Medical Career Support Center, Tottori University Hospital, Yonago 683-8504, Japan
}

\section{A R T I C L E I N F O}

\section{Keywords:}

Spirometra erinaceieuropaei

Plerocercoid

Excretory/secretory product

Immunomodulation

Plerocercoid-immunosuppressive factor (P-ISF)

Cestode cysteine-rich domain (CCD)

\begin{abstract}
A B S T R A C T
A platyhelminth, Spirometra erinaceieuropaei, belonging to the class Cestoda, causes human sparganosis, and infection with its larva results in subtle inflammation in the body of its host. We previously reported the purification of a glycoprotein, plerocercoid-immunosuppressive factor (P-ISF) from the excretory/secretory products of $S$. erinaceieuropaei plerocercoids that may be involved in immuno-modification. We determined the sequence of P-ISF from the N-terminal and the internal 10 amino acids of P-ISF using degenerate PCR and 5'- and 3'-RACE methods. The putative gene encoding P-ISF was $1443 \mathrm{bp}$ long and the gene contained 10 exons and 9 introns in a genomic DNA of size 5205 bp. P-ISF consists of 480 amino acids including the N-terminal signal peptide sequence, and has two unknown domains,-cestoda cysteine-rich domains (CCDs) and a fibronectin type III domain between the two CCDs. All cysteine residues were conserved in the two CCDs, which shared $62 \%$ amino acid identities. Homologous analysis revealed that the CCDs were homologous with an unknown protein of Diphyllobothrium latum. To produce specific antibodies, we expressed recombinant P-ISF (rP-ISF) using wheat germ protein synthetic system. P-ISF was localized in the sub-cutaneous tissues and the parenchymal tissues of plerocercoids. Transcription of P-ISF was detected only in plerocercoid stage, but not in adult stage. Western blotting also showed a band in plerocercoide stage but not in adult. The rP-ISF did not suppress nitrite production in RAW 264.7 cells stimulated with LPS, and this might be due to lack of carbohydrate chains in the recombinant protein.
\end{abstract}

\section{Introduction}

Some excretory/secretory (ES) products of various parasites have been reported as immunomodulators in their hosts [1]. These include plerocercoid of Spirometra erinaceieuropaei, which infects humans and causes sparganosis $[2,3]$. The adult worms of $S$. erinaceieuropaei live in the small intestine of mammals such as dogs and cats, and its infectious larvae called plerocercoids are parasitic in frogs and snakes in nature, but they can live in the subcutaneous tissues of mice and hamsters experimentally $[4,5]$.

Plerocercoids of $S$. erinaceieuropaei are parasitic in tissues, but intense inflammatory response or the formation of granuloma is not observed in the infection sites in the host. Based on this phenomenon, we hypothesized that plerocercoids secrete an immunomodulatory substance to evade the immune response of their host.

The crude ES products from $S$. erinaceieuropaei plerocercoids has been found to inhibit the mRNA expression of inducible nitric oxide synthase (iNOS) [6] in mice peritoneal macrophage stimulated with interferon- $\gamma$ and lipopolysaccharide (LPS), known as the ligand of Tolllike receptor (TLR) 4, in vitro. ES products also suppress the mRNA expression of tumor necrosis factor (TNF)- $\alpha$ [7] and interleukin (IL)-1 $\beta$ [8] in LPS-stimulated RAW 264.7 cells by reducing the phosphorylation of extracellular signal-regulated protein kinase $1 / 2$ and p38 mitogen activated protein kinase without affecting nuclear factor- $\mathrm{kB}$ activity [7].

Osteoclasts, the monocyte/macrophage lineage cells, were formed from the myeloid cells through RANKL stimulation. Crude ES products suppressed osteoclastgenesis. Next, we purified a glycoprotein using

\footnotetext{
*Corresponding author at: Division of Medical Zoology, Department of Microbiology and Immunology, Faculty of Medicine, Tottori University, 86 Nishi-cho, Yonago 683-8503, Japan.

E-mail address: otsuki@tottori-u.ac.jp (H. Otsuki).
} 
anion-exchange and lectin affinity chromatography, and named it plerocercoid-immunosuppressive factor (P-ISF) [9]. P-ISF suppressed osteoclastgenesis, the gene expression of TNF- $\alpha$ and IL-1 $\beta$, and nitric oxide (NO) production in LPS-stimulated RAW 264.7 macrophages.

In this study, we obtained cDNA fragment of P-ISF through RT-PCR with the degenerative primer-pairs that were designed based on the $\mathrm{N}$ terminal and the internal 10 amino acid sequences of the purified P-ISF. Next, we cloned the full-length cDNA using $5^{\prime}$ - and 3 '-rapid amplification of cDNA end (RACE) methods and determined the gene structure of P-ISF. We found that the deduced amino acid sequence of P-ISF was partially homologous to unknown proteins in Diphyllobothrium latum.

We speculated that the protein moiety of P-ISF might be necessary for exerting an inhibitory effect, because in a previous study the inhibitory effect of RANKL-stimulated bone marrow cells on osteoclastgenesis disappeared following protease treatment of P-ISF [9]. Next, we produced the recombinant P-ISF (rP-ISF) using a wheat germ protein synthetic system [10]. We examined the inhibitory activity of rP-ISF on NO production in RAW cells and we obtained an antibody for rP-ISF in mouse. We then investigated the localization of P-ISF in plerocercoid and the expression levels of P-ISF in the different growth stages of this parasite.

\section{Materials and methods}

\subsection{Parasites and animals}

Plerocercoids of $S$. erinaceieuropaei were collected from a species of snake (Elaphe quadrivirgata) in Shimane Prefecture, Japan, and stored for over 6 months in the subcutaneous tissues of Syrian hamsters aged 8 weeks or specific pathogen free (SPF) female C57BL/6 mice aged 68 weeks. The 8-week-old SPF female BALB/c mice were used to obtain serum antibody. All the mice and hamsters were purchased from Japan SLC, Inc. The adult worms of $S$. erinaceieuropaei used in the comparative study of isozyme patterns [11] were stored at $-80{ }^{\circ} \mathrm{C}$ until use in this study. All experiments were approved and performed in accordance with the guidelines of Institutional Animal Care and Use Committee of Tottori University.

\subsection{Preparation of crude ES products and purification of P-ISF}

The crude ES products of plerocercoids and P-ISF were prepared as described previously [9]. The medium containing the ES products was centrifuged to remove insoluble debris, and dialyzed against $25 \mathrm{mM}$ Tris- $\mathrm{HCl}$ (pH 7.4). This was concentrated using Amicon Ultra-15 Centrifugal Filter Units (Nihon Millipore Ltd., Tokyo, Japan), and subsequently sterilized with a $0.22 \mu \mathrm{m}$ filter (Nihon Millipore Ltd). Hereafter, these products are referred to as crude ES products. A glycoprotein, PISF, was purified from crude ES products using MonoQ HR 5/5 anionexchange (Amersham Biosciences Corp., Piscataway, NJ), RCA120Agarose (Seikagaku Corp., Tokyo, Japan), and WGA-Agarose columns (Seikagaku Corp.).

\subsection{DNA base sequence determination}

\subsubsection{A part of amino acid sequence analysis}

The purified P-ISF was electrophoresed on SDS-polyacrylamide gel and transferred onto PVDF membrane. The membrane and gel were stained with Coomassie Brilliant Blue, and the portion of the gel containing P-ISF was cut out from the SDS-PAGE gel and used as the sample for amino acid analysis. We obtained the N-terminal and a part of internal amino acid sequence analyses from APRO SCIENCE, Japan, and each ten amino acid sequence of P-ISF was obtained using Procise 494 cLC protein sequencer (Applied Biosystems, Foster, CA, USA). The Nterminal and internal amino acid sequences were estimated as Asn-GluAsp-Gly-Ile-Gly-Phe-Tyr-Asp-Leu, and Ile-*-Gly-Ser-Phe-Asp-Thr-PheAsn-Lys (*: no significant amino acid), respectively.
Total RNA from plerocercoids was extracted using an ISOGEN (Nippon Gene, Tokyo, Japan), and was reverse-transcribed into cDNA using M-MLV Reverse Transcriptase (Takara Bio., Shiga, Japan) and random primers (Takara Bio., Shiga, Japan). The degenerative primerpairs were designed based on the amino acid sequences. The cDNA was amplified using a sense primer (5'-AAC/T GAA/G GAC/T GGI ATI GGI TTC/T TAC/T GAC/T TT- ${ }^{\prime}$ ) and an antisense primer (5'-TTA/G TTA/G AAI GTG/A TCG/A AAI GAI CC-3'). Both ends of cDNA were extended with RACE [12,13]. 5'- and 3'-RACE were performed using SMART RACE cDNA Amplification Kit (Clontech, Mountain View, CA, USA) and each gene specific primer (GSP1:5'-AAT TGT TCA TCT GTG GCA TAT AGA CTC G-3', and GSP2:5'-TCT ATT GTA ATG CAT GGC TCC ATT TCA C-3') according to recommended protocol. These PCR products were cloned into pGEM-T Easy vector (Promega, Madison, WI, USA) and sequenced using Big Dye Terminator v3.1 Cycle Sequencing Kit and ABI PRISM 3130 sequencer (Applied Biosystems).

\subsubsection{Genome DNA sequence analysis}

To determine genome DNA base sequence, genome DNA was extracted from plerocercoids using QIAmp DNA mini Kit (QIAGEN, Hilden, Germany). Next, P-ISF gene was divided into three parts and amplified using the primer-sets that were designed based on the cDNA sequence as follows:

Fragment N: (sense) 5'-ATG TGC ACC GAC ATG TCG AGT C-3'/ (antisense) 5'-CCC ATT TAT GGT ACC TGG CTT G-3'.

Fragment mid: (sense) 5'-ACA GTG TAC TTC TGC CCT CGT G-3'/ (antisense) 5'-CAG CCT TTG CCT TTG GTT TCG TC-3'.

Fragment C: (sense) 5'-GCA TGA AGA GTG TGA ATT TGT GC-3'/ (antisense) 5'-TTA CGC ACG TGA CGT GCA ATT TTT G-3'.

All the fragment was cloned identically. The three fragments were sequenced using universal primers T7P and M13R. Additionally, the fragment mid was sequenced using the following four primers:

(sense inside1) 5'-AGC CAG GTA CCA TAA ATG GG-3'/(antisense inside1) 5'-CAC AAA TTC ACA CTC TTC ATG-3'/(sense inside2) 5'-CAT GCC TCA GGT ATC TTG-3'/(antisense inside2) 5'-GAT GAA ATA TTT GTA CGC CTG-3'.

\subsection{Expression and purification of rP-ISF}

Three PCR products, P-ISF (1-3), were amplified from cDNA using KOD Plus DNA polymerase with the following primers:

P-ISF (1): 5'-GTC GAC AAC GAA AAA GGA ATA GGA T-3' ${ }^{\prime} / 5^{\prime}-\underline{G T C}$ GAC tta CGC ACG TGA CGTG-3'.

P-ISF (2): 5'-GTC GAC AAC GAA AAA GGA ATA GGA T-3'/5'-GTC GAC tta CAA CTG ATA GAC TGG TAC TG-3'.

P-ISF (3): 5'-GTC GAC AAC GAA AAA GGA ATA GGA T-3'/5'-GTC GAC tta AAG TCC TGA CAT GCA AC-3'.

The stop codons are shown in lower case letters and the Sal I restriction enzyme sites are underlined. P-ISF (1) contains full-length cDNA except signal peptide sequence, P-ISF (2) contains cestoda cysteine-rich domain (CCD)1 and fibronectin type III domain (FN3), and P-ISF (3) contains CCD1 alone. These PCR products were inserted in Xho I site of the pEU-E01-GST-N2 plasmid vector, and they were expressed using the wheat germ cell-free protein synthesis system (Protemist DT; CellFree Sciences, Matsuyama, Japan). Recombinant proteins were captured using a glutathione column, washed, and eluted with glutathione elution buffer. Protein synthesis was confirmed by SDS-PAGE and Coomassie Brilliant Blue protein staining.

\subsection{Antisera}

The specific antisera were collected from mice immunized with each rP-ISF. Two BALB/c mice were injected with $25 \mu$ g of purified rP-ISF (1)-(3) and an equal volume of complete Freund's adjuvant. Subsequently, the mice were boosted with the same dose of incomplete adjuvant three times at 21-day intervals. The pre-immunized serum 
samples from naïve mice were used as negative control. To collect the sera that contain naturally acquired antibodies against whole plerocercoids, two mice were intraperitoneally infected with 10 plerocercoids. These mice were euthanized at 4 weeks post infection, and the serum samples were collected.

\subsection{Enzyme-linked immunosorbent assay}

The 96 well assay plates were coated with rP-ISF $(0.25 \mu \mathrm{g} / \mathrm{ml})$ or crude ES product $(0.25 \mu \mathrm{g} / \mathrm{ml})$ in $50 \mu$ lof borate buffer $(\mathrm{pH} 8.9)$ at $4{ }^{\circ} \mathrm{C}$ overnight. After blocking with $5 \%$ skim milk in PBST at room temperature for $1 \mathrm{~h}$, serial diluted antisera from 1:400 to 1:25,600 in PBST were added and incubated for $1 \mathrm{~h}$ at room temperature. HRP-goat antimouse IgG diluted at 1:1000 was sequentially added and incubated at room temperature for $1 \mathrm{~h}$, and was stopped with $100 \mu \mathrm{l} /$ well of $0.1 \mathrm{M}$ citrate buffer. After the final wash, the reactions were detected using 2-2'-Azinobis (3-ethylbenzothiazoline-6-sulfuric acid ammonium salt). Optical density (OD) values at $405 \mathrm{~nm}$ were determined with a microplate reader (Tecan Japan, Kanagawa, Japan). All samples were analyzed in duplicates.

\subsection{Western blot analysis}

\subsubsection{Preparation of the crude extract of plerocercoids and adult worm}

A roughly crushed worm, $3 \times$ SDS buffer, and double distilled water were mixed in the ratio of 1:1:1 and were homogenized using $1 \mathrm{ml}$ of syringe and $24 \mathrm{G}$ needle. They were centrifuged at $15,000 \mathrm{rpm}$ and $4{ }^{\circ} \mathrm{C}$ for $30 \mathrm{~min}$, and the supernatants were used as crude extracts.

\subsubsection{Western blotting}

The crude extracts of plerocercoids and adult worm, crude ES product, and rP-ISF were separated using SDS-PAGE (5-20\% gel) and transferred onto PVDF membrane. The gel was also stained with Coomassie brilliant blue to check that protein loading amount is equal. The membrane was blocked with 3\% $(w / v)$ skim milk and $0.05 \%$ Tween 20 in TBS for $1 \mathrm{~h}$ at room temperature. Next, the membrane was incubated overnight at $4{ }^{\circ} \mathrm{C}$ with mouse anti-rP-ISF (1) sera $(1: 25)$ or plerocercoid-infected mouse sera $(1: 25)$. The membrane was washed and incubated with a secondary antibody $(1: 25,000)$ for $1 \mathrm{~h}$ at room temperature. The signal was visualized using Immobilon Western Chemiluminescent HRP Substrate (Millipore, Billerica, MA, USA) and LAS-4000 (GE Healthcare Japan, Tokyo, Japan).

\subsection{Immunofluorescence microscopy}

Plerocercoids were collected and fixed in $10 \%$ formalin and embedded in paraffin. Sections were cut to $5 \mu \mathrm{m}$ thickness, fixed on glass slides, deparaffinized with xylene, and washed. The sections were blocked with PBS-milk and incubated with mouse anti-rP-ISF (1) sera $(1: 25)$ or anti-GST fused protein sera as control (1:25) for $1 \mathrm{~h}$ at room temperature. The sections were detected using a secondary antibody (Alexa 488 conjugated goat anti-mouse IgG, 1:500) and DAPI (1:1000). The sections were examined under fluorescent and DIC microscope BX60 (Olympus, Tokyo, Japan) and photographed using a DS-Ri1 CCD camera (Nikon, Tokyo, Japan).

\subsection{Expression of P-ISF gene}

To investigate the expression of P-ISF gene in plerocercoids and adult worm, total RNA was extracted from plerocercoids collected from snakes or mice and adult worms collected from the dogs. Reverse transcription was performed with $1 \mu$ g total RNA using M-MLV Reverse Transcriptase and random primers (Takara Bio.) according to the manufacturer's instructions. The primers included the fragment $\mathrm{N}$ sense and fragment $\mathrm{C}$ antisense primers, which were used for genome DNA sequence analysis as described above. $S$. erinaceieuropaei glyceraldehyde-3-phosphate dehydrogenase (GAPDH: GenBank accession No. AB031067.1) was amplified using a sense primer (5'-ATG AAG CCC AAG GTT GGT AT-3') and an antisense primer (5'-CTA GTG GTC CTT GGA GAA CAT-3') as control.

\subsection{RAW cell culture}

The murine macrophage cell line RAW 264.7 was cultured in Dulbecco modulated Eagle's medium (DMEM) and 5\% heat-inactivated fetal bovine serum (FBS) at $37{ }^{\circ} \mathrm{C}$ in an atmosphere of $5 \% \mathrm{CO}_{2}$. The cells were seeded at $5 \times 10^{4}$ cell/well $(200 \mu \mathrm{l} /$ well $)$ in 96 well plates for $12 \mathrm{~h}$. The culture medium was replaced with DMEM, supplemented with $5 \%$ FBS and with PBS (control), $5 \mu \mathrm{g} / \mathrm{ml}$ crude ES products, $10 \mathrm{ng} /$ $\mathrm{ml} \mathrm{P-ISF}, 10 \mathrm{ng} / \mathrm{ml}$, or $100 \mathrm{ng} / \mathrm{ml} \mathrm{rP-ISF}$, and with 5\% serum (anti-GST sera or anti-P-ISF (1)-(3) sera). The cells were incubated for $15 \mathrm{~h}$, and the culture medium was replaced with DMEM and $100 \mathrm{ng} / \mathrm{ml}$ LPS (from Escherichia coli 111:B4). After 24 h, the supernatants were collected for the measurement of nitrite.

\subsection{Nitrite assay}

The concentration of nitrite in the culture supernatant was measured as described previously [7]. In brief, $95 \mu \mathrm{l}$ of supernatant was added to a $96-$ well plate followed by $95 \mu 1$ of Griess reagent. After $15 \mathrm{~min}$ incubation, the absorbance was measured at $540 \mathrm{~nm}$ and nitrite concentration was determined with reference to a standard curve using sodium nitrite.

\subsection{Statistical analysis}

Data were analyzed using Graph Pad Prism 7 software. The nonparametric data were analyzed using one way analysis of variance (ANOVA).

\section{Result}

\subsection{Cloning of P-ISF gene and genome structure determination}

To clone P-ISF coding sequence, we analyzed P-ISF using Procise 494 cLC protein sequencer (Applied Biosystems). The results of this analysis revealed that each 10 amino acid sequence of $\mathrm{N}$-terminal and a part of internal amino acids of P-ISF as Asn-Glu-Asp-Gly-Ile-Gly-PheTyr-Asp-Leu and Ile-*-Gly-Ser-Phe-Asp-Thr-Phe-Asn-Lys, respectively (*: no significant amino acid; Fig. 1A, shown with underlines).

Using the protein-sequencing result described above, we designed the degenerative primers of P-ISF, and $375 \mathrm{bp}$ cDNA fragment was obtained by RT-PCR. Next, we cloned the rest of the full-length cDNA of P-ISF using 5'- and 3'-RACE methods. The predicted open reading frame (ORF) of P-ISF cDNA comprised 1443 bp and encoded 480 amino acids with the signal peptide of $\mathrm{N}$-terminal consisting of 26 amino acid residues.

The P-ISF ORF consisted of two cysteine rich regions and one fibronectin 3 domain between them. These cysteine rich regions were unique and had no similarities with known molecules; they were named as cestoda cysteine-rich domain (CCD). The $\mathrm{N}$-terminal side CCD1 (27-208 amino acids), C-terminal side CCD2 (299-480 amino acids), and FN3 located at 209-298 amino acids between the two CCDs were determined. The detailed information about nucleotide and amino acid sequences are presented in Fig. 1A. Each of CCD1 and CCD2 contained 16 cysteine residues, and these residues were conserved between the two CCDs (Fig. 1B), which shared $62 \%$ amino acids sequence identity. Multiple N- and O-glycosylation sites were predicted (Fig. 1B).

Genomic DNA amplification by PCR and sequencing showed that the P-ISF gene contained 10 exons and 9 introns in approximately $5.2 \mathrm{~kb}$ of genomic DNA (Fig. 1B). 
A 1 äGTGCACCGACATGTCGATCTTCGCTGGTTGATCCTGCTTCAACGTTPACCCTTCTGGCTGCTCTAGCGTTTgtaagttgtgcaatttgcttcaatacccacctgctctaattgct

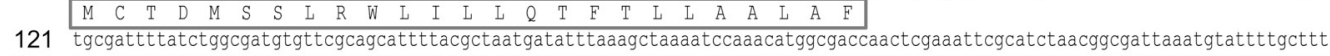
241 tagCGAACGAAAAAGGAATAGGATTCTATGACCTGTGCCAGGGGGAACATACAAATTGTTCATTTGTGGCCTATAGACTGGAAGATAAAACCAAGGGCAACGGTTGCCGTGTTGCGTGTG A $\quad \begin{array}{llllllllllllllllllllllllllllllllllllllllll}N & E & K & G & I & G & E & Y & D & L & C & Q & G & E & H & T & N & C & S & E & V & A & Y & R & L & E & D & K & T & K & G & N & G & C & R & V & A & C\end{array}$

361 GAAGGCAACGAGgttcgccagtttatctttgtctgctccaactacattcacatgtggcctggccatttcatatttatcgcaaactcccctgttcttaattgatggttttgtgaacagcaa $G \quad R \quad Q \quad R$

481 attcttcctcgttttagGGGAGCTAAATGAGACCATGACAGTGTACTTCTGTGAGGAACGCAACCAGACATGCAAACCGTACGCAGTCTATGGAAGAGAGAGATATGCCGGGAACTTTGA

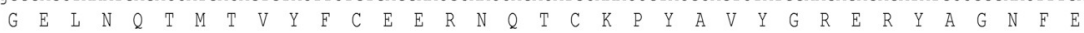

601 GCGGCACGTCAAGTGCGTGCAAACATGCCACTTTGTCGGAAGAGgtaagaagcctccttcactctcagccatttgagtaattatctcctgatagcattcccaattctttacaatactgct $\begin{array}{lllllllllllllllllllll}R & H & V & K & C & V & Q & T & C & H & F & V & G & R\end{array}$

721 gcggatgtgtctgcataagacatgcatccttctcgaaaacagacaaaaataaccaatgtacgcaaaatccttttgatttttagCTGAACATAGCTCCCACGATGACTTCGAAATATGTTC

A $l \begin{array}{llllllllllllllll} & \mathrm{S} & \mathrm{S} & \mathrm{S} & \mathrm{H} & \mathrm{D} & \mathrm{D} & \mathrm{F} & \mathrm{E} & \mathrm{I} & \mathrm{C} & \mathrm{S} & 130\end{array}$

841 CAACGAACTGGAGCCATGCAGTACAATAAGGAAGATAAATGGATCCTTTGATACCTTCAATAAATGCGCTTTTCAATGCGATGGCTGGCCAAAACgtgagttttgctaagtgtgctatta

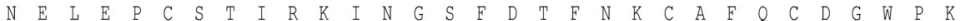

961 tcgttaagggcaagtcaattccaaggccttgttgatgttgagcatggttttttcttaaagTGGAAAACAGCGTTGAGGACACAGTGTACTTCTGCCCTCGTGAGGGTCATTGTGAGGAT $\begin{array}{lllllllllllllllllllllllll}L & E & N & S & V & E & D & T & V & Y & F & C & P & R & E & G & H & C & E & D\end{array}$

1081 GCCACATTCTTCATCAAGCCAGGTACCATAAATGGGTTCGAGCAGTTGATGAGCTGCTTCAACGGTTGCATGTCAGgtgagatcttgcatttaccattcaaaattttaaataatg tgtaa $\begin{array}{llllllllllllllllllllllllllllllllllllll}A & T & F & E & I & K & P & G & T & I & N & G & F & E & Q & L & M & S & C & F & N & G & C & M & S\end{array}$

1201 aatgacgaaagttaaaacagcgaagctcataaaaaagtgttttatctaaatgacgactatatacaaattgtagatgttcccggcatttaaattctgctgtacagtaatatcaatctgttc

1321 agttgatagaaactattggtgaagcattagagcacgcgagacaaaccgccatcgattcaagtcactgtcttttaatgtgcacactgaaactatctatatctcggagatatttgtggcgt

1441 ggctcactaagctattgtatgcaacgtaagctactgactcgtcaaatgctgatgtgatgtctccatgttgttgccttcacgtctttaattgacgcaaactttgctgacatatctagtatt

1561 ttgtatacgctaaaaagttctaatagagaataagtattaacggctaagcttgattttgcgcgagttttttcccgatctgttttaagtaaggagtgcattggatgtggaagacagtacaaa

1681 atgacggtatggtcacgcatgcctcaggtatcttgcgttttgttggtgagaacctaaagcacagccatagacaaacacggtgatttatgtccggaaatcctgcacctgtacgtccacatt

1801 gcgttatgtgaacgccatgcccacgtgaaatgcgaacagacttgctcgcggcgagggctgaccgcattattctctaaacctatcgtgtctaattgttttctttatcggataaacatctag

1921 cttgactatatgatgacgaaatctcccttgcatatatcgccaaatattgaagcacctgtcgaagcatattcacattcacagttttggtaattgatattgaataagccgcgtaaaagcta

2041 attattttctcagttaagggctgtcagcattcgtatttcaacgcactttctagcatcttaaccaggccaaccaagtcgtccggtattttttcaaggtaattaataaaagtcagcatgtaa

2161 ttggccatacgcgaccaaaagtgtgtttatttgaagggaagttattttttctggcocgtatgcggagaacgtccagtatagaagtctacaaacttgtcattcaaaggaaagatagcatgt

2281 ggcgaatatcggctggtcaacaatttacttccgtataacgtagcgataacagacgacgaacaagtgcccaaaattcggcagtcgaatgctgtaagacacatcattcattttgcattttta

2401 agGACTTGTGCCAACAGAGTTTACTGCGACGGCTGTTAATTCGTCCGCCATCCGTGTGACCTGGAAGAAACCCCGCCGGTCTGGTGAACTAGGCGACAAGTACGAACTTATCCTACGCAA

2521 CAGCACGCACGAGGATGAATTTAACCTACGTGTGACTGAGGACATAATTGCGGACCTTGAGCCTTCTACCACTTACAACTTGACCGTGCGGGCTTTCTGGAGCAATAACACCCCAGTCGA

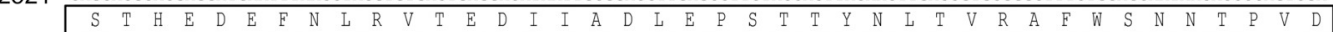

2641 TGCGGTCGCATTCACATCAGTGACAACGCAGCCACGGGgtacgctaaaacttaattgtatttactgttccatgcttccacgCccacaagaacgttcacgatgtgcgcgtccttaatgtct \begin{tabular}{llllllllllllll}
$A$ & $V$ & $A$ & $F$ & $T$ & $S$ & $V$ & $T$ & $T$ & $Q$ & $P$ & $R$ \\
\hline
\end{tabular}

2761 agtgctgtatcctgcgcccaagaggggcaatcgatagatatcctccccgatgctaatcggcctctaaagagtttcgccaaagtaagcactctccagagatgtggggtctcaagtttgata

2881 agcattcttgcaatgatacqctgatatttcaaataatgcatgcqtttagtacacaaqcacccqcatttacaaaggcattattctccaaaactttacggctgccttctcagqgcaaaatcc

2881 agcttttccttcttcattatgtgctggctaaacgcagctgcataagaggataaggaccaatagagatgattaacggaagccatcccttaatcgtgcttttaaatatcaaaattatcactt

3121 atgagtcacttaggagccttaaattatgctgatcaataattcacctaagcatgtttggcggtatttcggcatcctactaatgccaactctttttttagttccaagataaaatggccaatt

3241 tgttcaggcatctctggttgcaaatacgcatgttcccgtatcagctagtatttagccttgaatgatatttttacactagtaaggaataatttgaggattgcttttctcttttatatag

3361 ggtggggtccttttagttcgcgtctggttatactggacacggatatacaagtgaatttactgggaaatattaaagtagtccgcgtttaaaatggcgtattgtagtacctgtacgcaagga

3481 atattgtgtctaagtcagttatctgatttatctctcacatgcttttcatttcccoctcttctgctcatcggagtaaccttttacttgtggcccaataattatcattcgtcaaaaatgtt

3601 ctcgtacaggcgtacaaatatttcatcgtgtctcgatgaccttatacatgcaaacaatttttaatatcaattgtaaacatatggtgcacttaggacattttccagaaaattccgtgttcg

3721 tcatgtcagctgtgtttataattcacattttgggctacttgcatcacttatgaaatttcaaaaccttaaacatactatatagatgtactggtcatttctattaaatcaattctctttatt

3841 ggatgaagtggataaaaaccatctattggaaaacctgtaggtttaatccgagaaatcgcatatcagacgtagttgttttgatgaggctgtagatttcaaatcctccaatgccaagagaag

3961 acaggctggtctttaatgcgcatctcaaattgacttttattttccattcgtaaaatgtaagttattcgacgtaaatttccggatgaactatattgctttgttactggagtgcaattaatt

4081 gcaagcgtatgaaaaaacgctttgtagatgatcgaaacctgcagacaaatcgatagcggactatgcaaacgtcgcagcattcatcagctttacttattttactataatttatccaatctt

4201 agggggattttgattgtactcagttaattgcccgcaggaattgcatttaggatacgcagagagaaaatctaaaaaagcgtatctactcaatagcttcctctgattttcaatttcattcat

4321 tttgtttttaacagAGGAGGACACAGTACCAGTCTATCAGTTGTGCAAAAAGGAGCATGAAGAGTGTGAATTTGTGCCCTACAGAGAAAAAGACGAAACCAAAGGCAAAGGCTGCCGTGA

4441 AGCGTGTGCAAB E E A $C$ A $\quad K \quad$ Q

4561 aagcaacacattcttccttgtttcctagGTGGACTAAATGAGACAATGACAGTATACTATTGCGATGAACCCAACGCAAAATGCACGCCATACGAAGTTIATGGACGAGCGGGATATGCC

$\begin{array}{llllllllllllllllllllllllllllllll}G & G & L & N & E & T & M & T & V & Y & Y & C & D & E & P & N & A & K & C & T & P & Y & E & V & Y & G & R & A & G & Y & A\end{array}$

4681 GAGAACTTTGAGAGGCACTTTAAGTGCGTCAGTCTGTGTCACCTTGTCGGGAGAGgtaaattttctccttgctcatgaatattttgtgcgacgccatccttctggctttctaaatacgtt $\begin{array}{llllllllllllllllllllllllllll}E & N & F & E & R & H & F & K & C & V & S & I & C & H & L & V & G & R\end{array}$

4801 gacagtggcaggcacgacttatacgcaattatttccccgtttttagTTCAACATAGCGCCTACAATGATTTTGAAATGT GCTCTGGCGAACTGGAACCATGCGTGAAAATAGAGAAAATA

$\begin{array}{lllllllllllllllllllllllll}V & Q & H & S & A & Y & N & D & F & E & M & C & S & G & E & L & E & P & C & V & K & I & E & K & I\end{array}$

4921 AACGGAACATTTGATGCTTTCAATAAATGTGCTTTTCAATGCGACGGCTGGCCAAATCgtgagttcatatttttgtacagtaatttttaataactaatcgtataaagttattgttaatgt

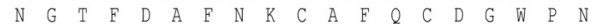

5041 tacggattttttcactttaagTTGAAAACACCTTGGAGGACACAGCATTTTTCTGCCCTCATGAAGGATATTGTCAGGATGCCTATTTCTTTAGCAAGCCGGATGACAAAAATGGATTT $\begin{array}{lllllllllllllllllllllllllllllllllll}L & E & N & T & L & E & D & T & A & F & F & C & P & H & E & G & Y & C & Q & D & A & Y & F & F & S & K & P & D & D & K & N & G & F & 466\end{array}$

5161 GAAAGCCTGATGACCTGCCTCAAAAATTGCACGTCACGTGCGTAA

E

\section{P-ISF}
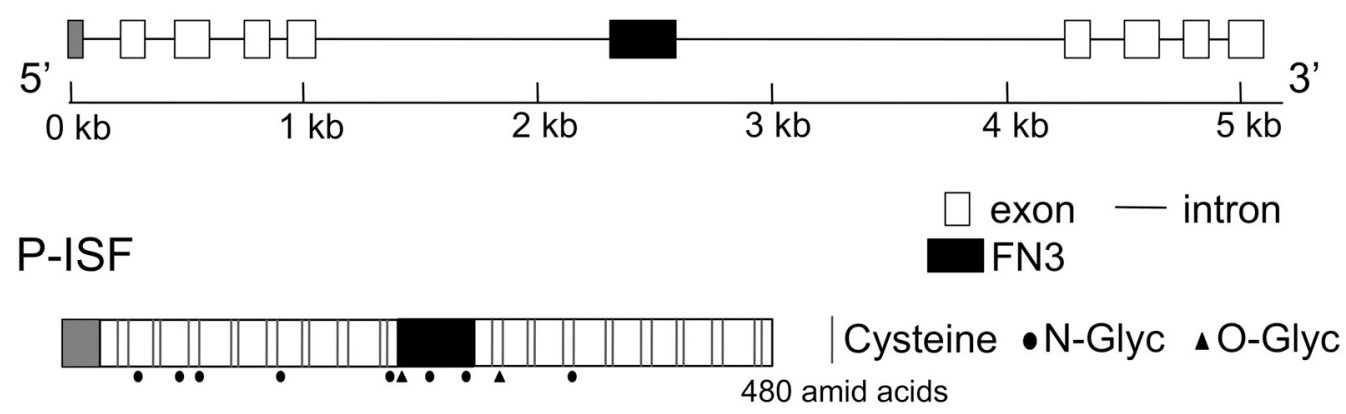

(caption on next page) 


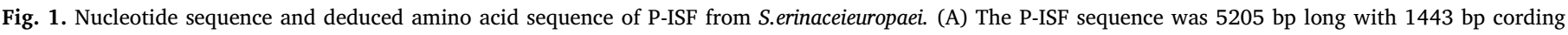

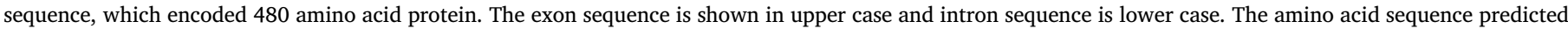

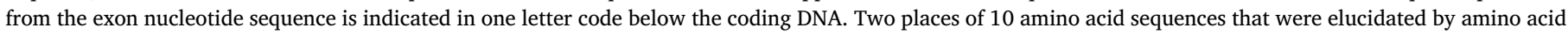

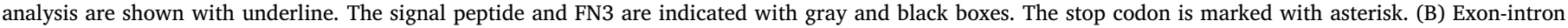

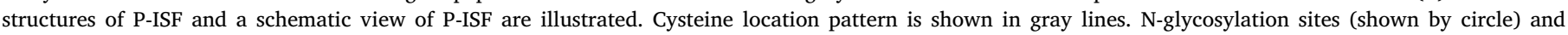
O-glycosylation sites (shown by trigone) were predicted. Dark boxes indicate FN3.

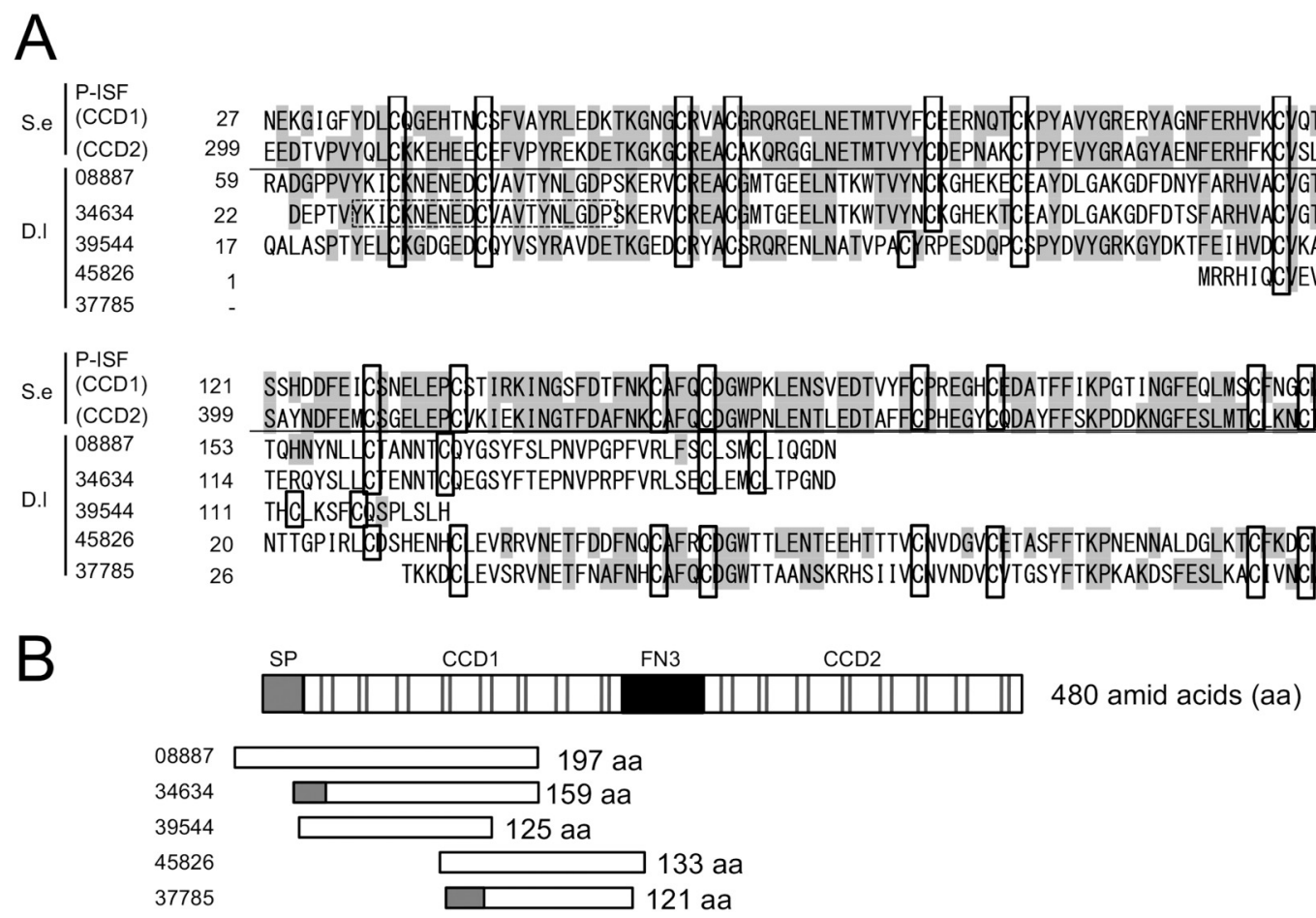

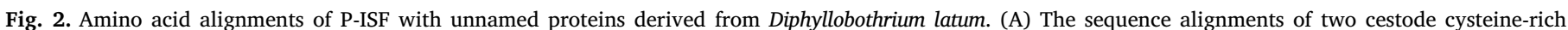

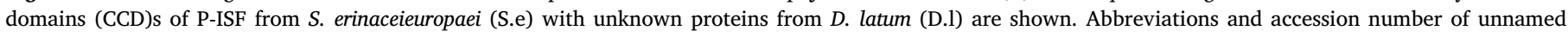

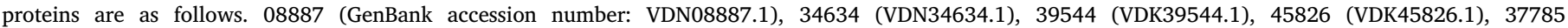

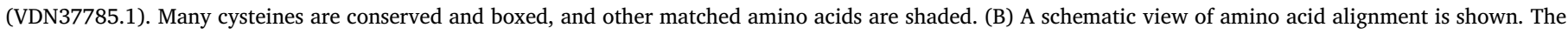

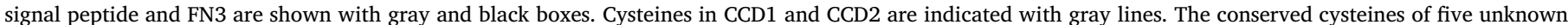
proteins from $D$. latum are aligned with cysteines of CCD1 of P-ISF.

3.2. Alignment of amino acids of CCDs between $S$. erinaceieuropaei and D. latum

Since P-ISF is a novel molecule, we attempted to detect the gene from the genome database. Through the homology search of the nucleotide sequence using BLAST, three fragments from S. erinaceieuropaei database showed $>92.5 \%$ identities with P-ISF but these were scattered among different contigs [14]. No homologs with high identities in other organisms except $S$. erinaceieuropaei were detected. The BLASTx analysis showed that parts of the deduced amino acid sequence of P-ISF were homologous with five unknown proteins of Diphyllobothrium latum. These proteins contained 7-12 cysteine residues that were well conserved with P-ISF but they were shorter than P-ISF (Fig. 2A, B).

\subsection{Expression and purification of rP-ISF using wheat germ system}

Three different regions of P-ISF, (1) full-length, (2) CCD1 and FN3, and (3) CCD1 alone, were amplified from cDNA (Fig. 3A), sub-cloned into GST-fused pEU-E01-MCS vector, and expressed using the wheat germ cell-free protein synthesis system. Recombinant proteins were expressed in soluble fraction and purified through glutathione sepharose $4 \mathrm{~B}$ affinity chromatography. These three rP-ISF (1-3) with the estimated molecular sizes were confirmed on SDS-PAGE gel (Fig. S1).

Mice were immunized using these purified rP-ISF, and all sera detected the three rP-ISF (1)-(3) by ELISA with anti-rP-ISF (1)-(3) sera of mice, respectively (Fig. 3B). Native P-ISF in ES products was also detected using these sera (data not shown).

\subsection{Detection of P-ISF from ES product and parasite tissue from $S$. erinaceieuropaei plerocercoid}

Using these specific antisera against rP-ISF, western blot analysis was performed to detect native P-ISF from ES product and parasite tissue from plerocercoides. An approximately $75 \mathrm{kDa}$ band was detected in both ES and extracted samples. This is larger than the expected molecular size, and this may be due to its glycoprotein feature (Fig. 3C). The rP-ISF (1) was also recognized by the sera of plerocercoids-infected mice, and proved that antibodies are increased against secreted P-ISF in plerocercoid infected mouse (data not shown).

\subsection{Immmunolocalization of P-ISF in plerocercoid}

To identify the localization of P-ISF in plerocercoids, the sagittal sections and the cross sections of plerocercoids were examined under a microscope by fluorescent antibody staining with anti-rP-ISF (1) sera (Fig. 4). P-ISF was observed to be diffused in the parenchymal tissues of the whole scolex and body, and was accumulated in the subtegmental tissues. The tegument was not stained, but some fluorescent lines led to body surface from the parenchymal tissues through a tegument as shown with white arrowheads (Fig. 4A). The results indicated that P-ISF 
A

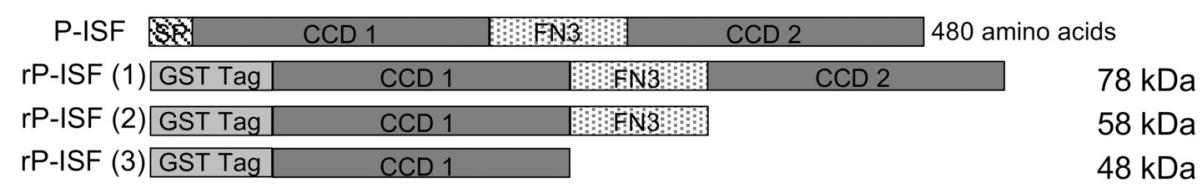

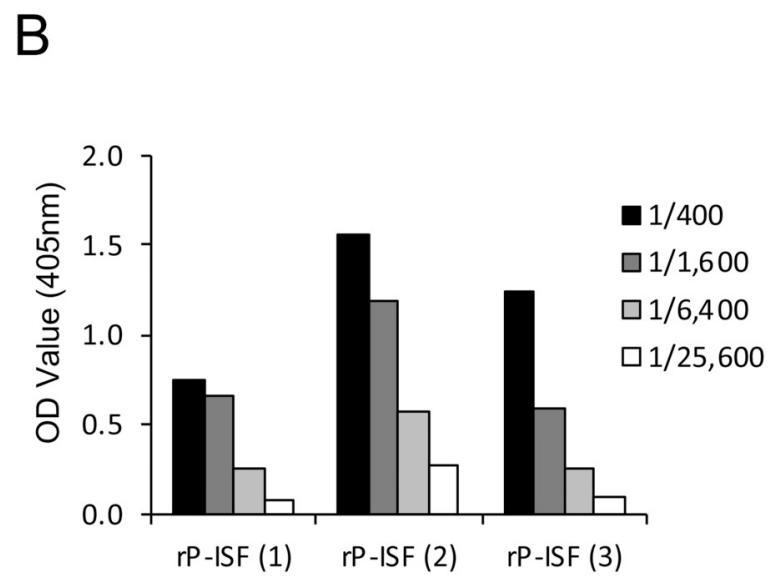

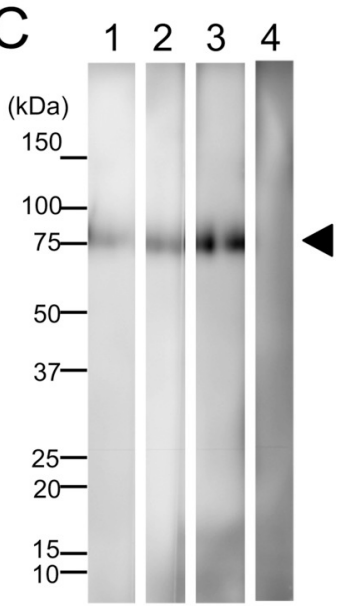

Fig. 3. The expression of rP-ISF and production of antisera. (A) Schema of P-ISF and GST-fused three different rP-ISFs, (1) full-length, (2) CCD1 and FN3, (3) CCD1 alone, were illustrated. (B) The rP-ISF (1)-(3) were recognized by serially diluted anti-rPISF sera of mice in ELISA. (C) Western blotting of native P-ISF in crude ES products. Lanes 1-4 were applied with the crude ES products $(7 \mu \mathrm{g})$. Lanes $1-3$ were probed with each mouse anti-rP-ISF (1)-(3) sera and lane 4 was recognized by the anti-GST fused protein sera as a negative control. was produced in the whole body of plerocercoids and was secreted into host.

\subsection{Growth stage specific expression of P-ISF}

The P-ISF mRNA expression was detected in plerocercoids from snakes and mice. However, it was not observed in adult worms from dogs even though GAPDH gene expression was detected at both stages (Fig. 5A). P-ISF protein was also examined in the supernatants obtained from the homogenates of plerocercoids and adult worm through western blot analysis as described above. It was confirmed that adequate quantity of protein has been loaded in both stage by SDS-PAGE. (Fig. S2). P-ISF protein was expressed at plerocercoid stage, but was not expressed at adult stage (Fig. 5B).

\subsection{The effect of $r P$-ISF on NO production in LPS-stimulated macrophages}

The crude ES products and P-ISF significantly suppressed NO production in LPS-stimulated RAW 264.7 macrophages, as reported in our previous study [9], but none of the three rP-ISFs inhibited NO production in LPS-stimulated macrophages (Fig. S3A). NO production was not changed with increasing concentration of rP-ISF. The recombinant protein itself did not activate macrophages (Fig. S3B).

\section{Discussion}

Previously, we purified a glycoprotein, which has an immunosuppressive effect on LPS-stimulated RAW 267.4 cells, from crude ES products of $S$. erinaceieuropaei plerocercoids and named it P-ISF [9]. In the present study, we obtained cDNA fragment through RT-PCR using degenerative primer-pairs that were designed based on 10 amino acid sequences located at the $\mathrm{N}$-terminal region and inside the purified native P-ISF. Furthermore, we cloned cDNA encoding the complete ORF of P-ISF using 5'- and 3'-RACE methods and analyzed the gene structure.

The coding region of P-ISF consisted of 1443 bp nucleotides encoding 480 amino acids. P-ISF had a signal peptide (1-26 amino acids) at the N-terminus of ORF, and this indicated that P-ISF was a secretory protein. This novel protein was characterized with two almost identical cysteine rich regions of unknown functions; therefore, we named them CCD. The N-terminal side CCD1 and C-terminal side CCD2 contained 16 cysteine amino residues and all the cysteine residues were completely conserved, and they shared $62 \%$ amino acid sequence identity. The P-ISF had FN3 between CCD1 and CCD2, and it was expected to have a complex structure.

No homologous nucleotide sequence to the two CCDs was found in any other organism using BLAST searches except $S$. erinaceieuropaei itself. However, BLASTx analysis showed that the partially deduced amino acid sequence of the two CCDs was closely related to five unknown proteins of Diphyllobothrium latum [15] and three unknown proteins of Schistocephalus solidus [16](date not shown). The locations of cysteine residues were almost conserved among these proteins, but the functions of these proteins remain unknown. Although Taenia solium, Taenia ovis, and Dictyocaulus viviparus have a protein including FN3 $[17,18]$, but no protein with cysteine rich domain connecting FN3 has been reported to date. We also analyzed the genomic sequence of $\mathrm{P}$ ISF based on cDNA results, and P-ISF exhibited a complex gene structure composed of 10 exons and 9 introns.

P-ISF was detected in both solubilized plerocercoid and a culture medium of plerocercoids through western blotting with an antibody against rP-ISF. It appeared as P-ISF was synthesized in plerocercoid and released in the host tissues around the parasite.

Following immunostaining with specific antibodies, P-ISF was found to be localized in the systemic parenchyma of the scolex and body portions, and it accumulated under the tegument. Because some fluorescent line were observed from the tegument bottom till the body surface, it was presumed that P-ISF is secreted through the secretory glands, which is similar to $23 \mathrm{kDa}$ cysteine protease (Se23kCP) secreted from the body surface of plerocercoids of $S$. erinaceieuropaei [19].

P-ISF expressions of both mRNA and protein were found only in plerocercoid stage regardless of the host, but were not found in adult stage in the intestine. This may be because an immunosuppressive 
A

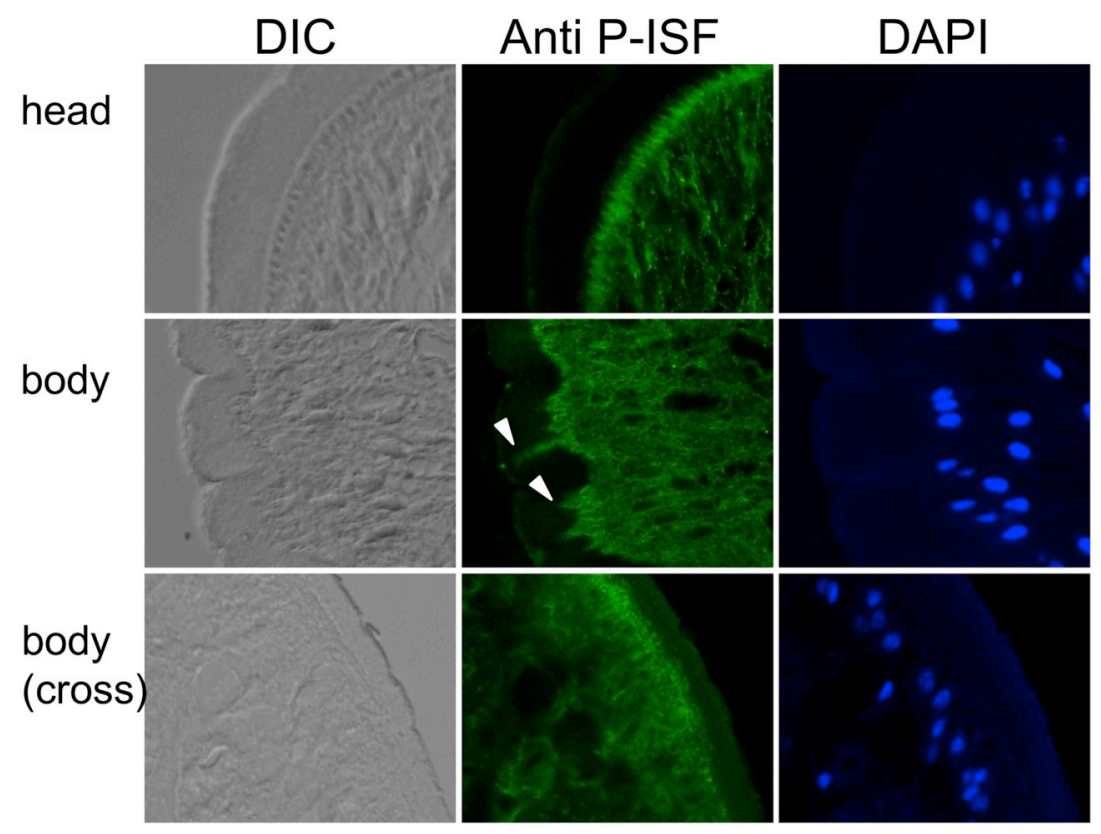

B

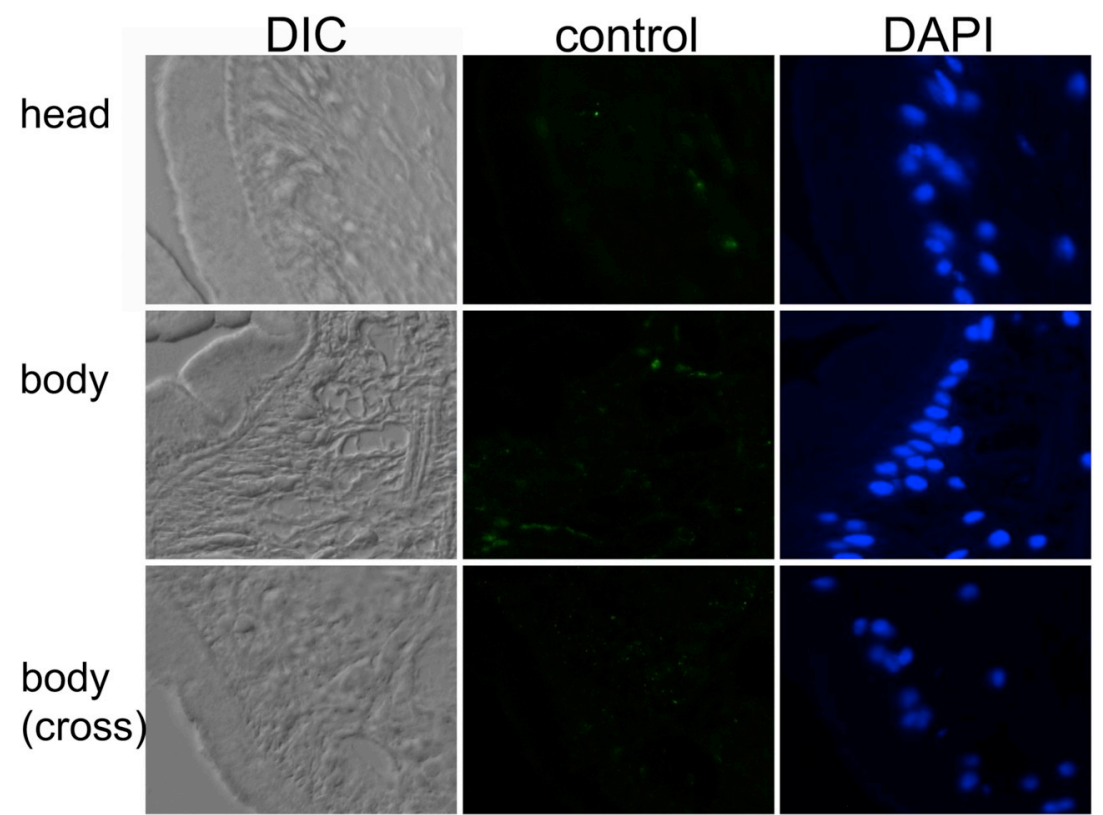

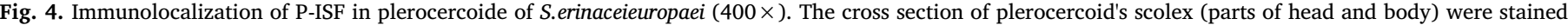

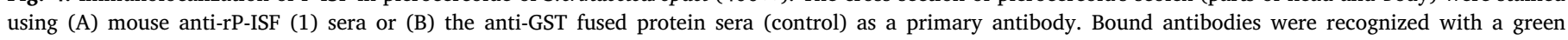
fluorescent-labeled secondary antibody. The sections incubated with DAPI (blue) for nuclear staining. DIC images are shown in the left-hand column.

product such as P-ISF in the adult worm was less important in the small intestinal lumen of the host with less exposure to immune responses than that in plerocercoids seen in host tissue with highly accumulated immune responses. S. erinaceieuropaei cysteine protease (SeCP) has been reported as a plerocercoid stage-specific protein similar to P-ISF [20]. However, S. erinacei casein kinase I (SeCK I) is expressed at both adult stage and plerocercoid stage [21].

rP-ISF expressed with the wheat germ cell-free system did not exert a suppressive effect on nitrite production in LPS-stimulated RAW cells, and the antisera elicited with these recombinant proteins could recognize native P-ISF in parasite sample. The following two reasons were considered to be responsible for this phenomenon. First, protein folding could differ from native protein, because P-ISF contained many cysteine residues to be folded correctly, even when wheat germ cell-free system was used. Second, the carbohydrate chain might be responsible for the physiological activity of P-ISF. A purified glycoprotein (P-ISF) from ES products strongly inhibited osteoclast formation from the murine bone marrow cells stimulated with RANKL and macrophage colony-stimulating factor. The inhibitory activity of P-ISF on osteoclastgenesis and the gene expression of calcitonin receptor and tartrate-resistant acid phosphatase disappear after trypsin-treatment [9], which suggests that the protein moiety of P-ISF is related to the inhibitory activity. However, three rP-ISF (1)-(3) were expressed using the wheat germ cell-free protein system, which did not glycosylate a recombinant protein; therefore, it might not inhibit NO production in LPS-stimulated RAW cells. Both the protein moiety and carbohydrate chains might be necessary for inhibitory activity on macrophages. To overcome this issue, other eukaryotic protein synthetic systems that can add carbohydrate chains would be adapted in our future study.

Parasitic helminthes achieve long-term survival in the host as they 


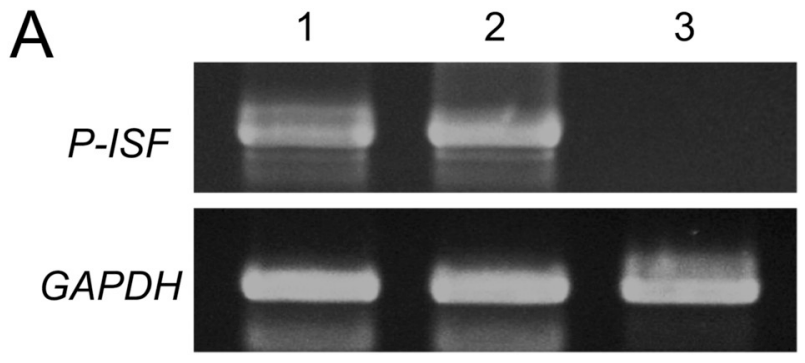

B

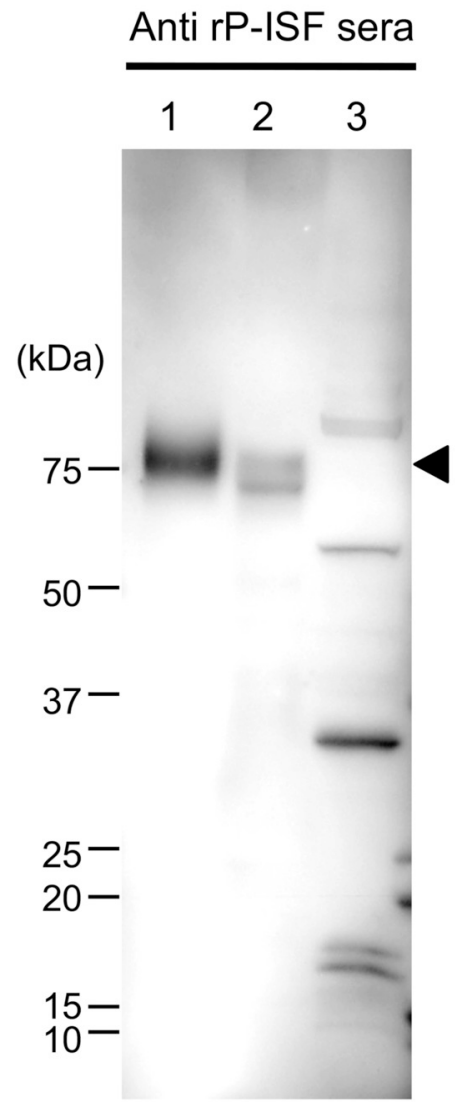

Fig. 5. Expession of P-ISF at different developmental stage of $S$. erinaceieuropaei. (A) The mRNA expression of P-ISF and GAPDH by RT-PCR is shown in the plerocercoids from snake (lane 1), from mouse (lane 2), and in adult worm from dog (lane 3). (B) The protein expression of P-ISF is examined by Western blotting. Crude ES products of plerocercoid (lane 1) and the crude extracts of plerocercoid (lane 2) and adult worm (lane 3) were electrophoresed and recognized by anti rP-ISF (1) sera. The crude ES product $(1 \mu \mathrm{g})$, the homogenized plerocercoids and adult worm(each $10 \mu \mathrm{l}$ of nine fold dilution of crude extracts) were applied.

have multiple mechanisms to suppress immune responses. Some helminth-derived immunomodulatory molecules have been reported previously [3,22], and two molecules, which suppressed the activation of macrophages, have been already cloned.

One such product is the $62 \mathrm{kDa}$ factor, ES-62, which is an abundant phosphorylcholine containing secreted glycoprotein of Acanthocheilonema viteae, the filarial nematode of rodents. A full-length cDNA for ES-62 corresponding to 474 amino acid residues has been cloned and identified as leucine aminopeptidase [23]. ES-62 modulates macrophages and dendritic cells by first binding with TLR4 on their surface [24]. Both ES-62 and P-ISF suppress mRNA expression of TNF- $\alpha$ in the LPS-stimulated macrophages and osteoclastgenesis from bone marrow cells with RANKL stimulation $[9,25,26]$. However, there is a difference between P-ISF and ES62 in their effects on NO production of macrophages. P-ISF suppressed NO production in LPS-activated macrophage, but ES-62 did not inhibit NO production $[9,25]$.

Schistosome mansoni cercarial ES also contains immunomodulator Sm16 that can inhibit TLR signaling in monocyte through a mechanism that does not depend on direct binding to TLR. The putative gene coding Sm16 is 500 bp with ORF of 117 amino acids that include the Nterminal signal peptide sequence, and this gene expression appears not to be stage-specific [27]. Sm16 has functional similarities with highly conserved microtubule-regulatory protein stathmin of vertebrates. Sm16 supposedly blocks NF- $\mathrm{kB}$ activation at very early stages of TLR signaling [28]. ES products from $S$. erinaceieuropaei do not inhibit LPSinduced nuclear translation of NF- $\mathrm{kB}$ but suppress the phosphorylation of MAPK in macrophages [7].

The suppressive mechanisms of P-ISF were different from those of helminth-derived immunomodulatory molecules such as ES62 and Sm16, and we reported a characterization and a function of a new immunosuppressive glycoprotein, P-ISF, in this study. As the crude ES products of $S$. erinaceieuropaei suppress IP-10 gene expression in LPS and/or IFN- $\gamma$ stimulated macrophages [29], P-ISF may reduce Th1-dependent immune reaction and may be effective in the treatment of autoimmune diseases as well as helminth-derived molecules previously reported $[26,30,31]$.

Supplementary data to this article can be found online at https:// doi.org/10.1016/j.parint.2020.102062.

\section{Funding}

This work was supported by a JSPS Grant-in-Aid for Scientific Research (C): grant No. 21590464.

\section{Declaration of Competing Interest}

All authors have declared no conflict of interest.

\section{Acknowledgements}

We thank the Research Center for Bioscience and Technology, Tottori University for technical assistance. We thank the volunteer people for sample collecting in the field.

\section{References}

[1] J.P. Hewitson, J.R. Grainger, R.M. Maizels, Helminth immunoregulation: the role of parasite secreted proteins in modulating host immunity, Mol. Biochem. Parasitol. 167 (2009) 1-11, https://doi.org/10.1016/j.molbiopara.2009.04.008.

[2] T.H.C. Tang, S.S.Y. Wong, C.K.C. Lai, R.W.S. Poon, H.S.Y. Chan, T.C. Wu, Y.F. Cheung, T.-L. Poon, Y.-P. Tsang, W.-L. Tang, A.K.L. Wu, Molecular identification of Spirometra erinaceieuropaei tapeworm in cases of human sparganosis, Hong Kong, Emerg. Infect. Dis. 23 (2017) 665-668, https://doi.org/10.3201/eid2304.160791.

[3] D. Tappe, L. Berger, A. Haeupler, B. Muntau, P. Racz, Y. Harder, K. Specht, C. Prazeres da Costa, S. Poppert, Case report, Molecular diagnosis of subcutaneous Spirometra erinaceieuropaei sparganosis in a Japanese immigrant, Am. J. Trop. Med. Hyg. 88 (2013) 198-202, https://doi.org/10.4269/ajtmh.2012.12-0406.

[4] O. Liu, M.-W. Li, Z.-D. Wang, G.-H. Zhao, X.-Q. Zhu, Human sparganosis, a neglected food borne zoonosis, Lancet Infect. Dis. 15 (2015) 1226-1235, https://doi. org/10.1016/S1473-3099(15)00133-4.

[5] S.-H. Lee, J.-S. We, W.-M. Sohn, S.-T. Hong, J.-Y. Chai, Experimental life history of Spirometra erinacei, Korean J. Parasitol. 28 (1990) 161-173, https://doi.org/10. 3347/kjp.1990.28.3.161.

[6] S. Fukumoto, K. Hirai, T. Tanihata, Y. Ohmori, D.J. Stuehr, T.A. Hamilton, Excretory/secretory products from plerocercoids of Spirometra erinacei reduce iNOS and chemokine mRNA levels in peritoneal macrophages stimulated with cytokines and/or LPS, Parasite Immunol. 19 (1997) 325-332, https://doi.org/10.1046/j 1365-3024.1997.d01-215.x.

[7] P. Dirgahayu, S. Fukumoto, K. Miura, K. Hirai, Excretory/secretory products from plerocercoids of Spirometra erinaceieuropaei suppress the TNF- $\alpha$ gene expression by reducing phosphorylation of ERK1/2 and p38 MAPK in macrophages, Int. J. Parasitol. 32 (2002) 1155-1162, https://doi.org/10.1016/S0020-7519(02) 00090-5.

[8] P. Dirgahayu, S. Fukumoto, S. Tademoto, Y. Kina, K. Hirai, Excretory/secretory 
products from plerocercoids of Spirometra erinaceieuropaei suppress interleukin-1 $\beta$ gene expression in murine macrophages, Int. J. Parasitol. 34 (2004) 577-584, https://doi.org/10.1016/j.ijpara.2003.12.006.

[9] Y. Kina, S. Fukumoto, K. Miura, S. Tademoto, K. Nunomura, P. Dirgahayu, K. Hirai, A glycoprotein from Spirometra erinaceieuropaei plerocercoids suppresses osteoclastogenesis and proinflammatory cytokine gene expression, Int. J. Parasitol. 35 (2005) 1399-1406, https://doi.org/10.1016/j.ijpara.2005.05.012.

[10] T. Tsuboi, S. Takeo, H. Iriko, L. Jin, M. Tsuchimochi, S. Matsuda, E.-T. Han, H. Otsuki, O. Kaneko, J. Sattabongkot, R. Udomsangpetch, T. Sawasaki, M. Torii, Y. Endo, Wheat germ cell-free system-based production of malaria proteins for discovery of novel vaccine candidates, Infect. Immun. 76 (2008) 1702-1708, https://doi.org/10.1128/IAI.01539-07.

[11] S. Fukumoto, T. Tsuboi, K. Hirai, C.K. Phares, Comparison of isozyme patterns between Spirometra erinacei and Spirometra mansonoides by isoelectric focusing, J. Parasitol. 78 (1992) 735-738, https://doi.org/10.2307/3283554.

[12] M.A. Frohman, M.K. Dush, G.R. Martin, Rapid production of full-length cDNAs from rare transcripts: amplification using a single gene-specific oligonucleotide primer, Proc. Natl. Acad. Sci. U. S. A. 85 (1988) 8998-9002, https://doi.org/10.1073/pnas. 85.23.8998.

[13] T. Ozawa, M. Kondo, M. Isobe, 3' rapid amplification of cDNA ends (RACE) walking for rapid structural analysis of large transcripts, J. Hum. Genet. 49 (2004) 102-105, https://doi.org/10.1007/s10038-003-0109-0.

[14] GenBank accession number: LN247998.1, LN363412.1, LN.100485.1.

[15] GenBank accession number: VDN08887.1, VDN34634.1, VDK39544.1, VDK45826. 1, VDN37785.1.

[16] GenBank accession number: VDM00388.1, VDM05095.1, VDM06344.1.

[17] C.G. Gauci, C.M. Jayashi, A.E. Gonzalez, J. Lackenby, M.W. Lightowlers, Protection of pigs against Taenia solium cysticercosis by immunization with novel recombinant antigens, Vaccine 30 (2012) 3824-3828, https://doi.org/10.1016/j.vaccine.2012. 04.019.

[18] C. Gauci, M.W. Lightowlers, Molecular cloning of genes encoding oncosphere proteins reveals conservation of modular protein structure in cestode antigens, Mol. Biochem. Parasitol. 127 (2003) 193-198, https://doi.org/10.1016/S0166-6851(03) 00005-7.

[19] D. Tsubokawa, T. Hatta, H. Maeda, F. Mikami, Y. Goso, T. Nakamura, M.A. Alim, N. Tsuji, A cysteine protease from Spirometra erinaceieuropaei plerocercoid is a critical factor for host tissue invasion and migration, Acta Trop. 167 (2017) 99-107, https://doi.org/10.1016/j.actatropica.2016.12.018.

[20] L.N. Liu, Z.Q. Wang, X. Zhang, P. Jiang, X. Qi, R.D. Liu, Z.F. Zhang, J. Cui, Characterization of Spirometra erinaceieuropaei plerocercoid cysteine protease and potential application for serodiagnosis of sparganosis, PLoS Negl. Trop. Dis. 9 (2015) 1-19, https://doi.org/10.1371/journal.pntd.0003807.

[21] L.N. Liu, H. Wang, P. Jiang, M. Wang, M.G. Xu, L.F. Wang, X. Qi, X. Zhang, J. Cui,
Z.Q. Wang, Molecular cloning and characterization of a Spirometra erinacei casein kinase I, Parasitol. Res. 114 (2015) 4737-4742, https://doi.org/10.1007/s00436015-4758-7.

[22] P. Adisakwattana, S.P. Saunders, H.J. Nel, P.G. Fallon, Helminth-derived immunomodulatory molecules, Adv. Exp. Med. Biol. 666 (2009) 95-107, https://doi. org/10.1007/978-1-4419-1601-3_8.

[23] W. Harnett, K.M. Houston, R. Tate, T. Garate, H. Apfel, R. Adam, S.M. Haslam, M. Panico, T. Paxton, A. Dell, H. Morris, H. Brzeski, Molecular cloning and demonstration of an aminopeptidase activity in a filarial nematode glycoprotein, Mol. Biochem. Parasitol. 104 (1999) 11-23, https://doi.org/10.1016/s0166-6851(99) 00113-9.

[24] H.S. Goodridge, F.A. Marshall, K.J. Else, K.M. Houston, C. Egan, L. Al-Riyami, F.Y. Liew, W. Harnett, M.M. Harnett, Immunomodulation via novel use of TLR4 by the filarial nematode phosphorylcholine-containing secreted product, ES-62, J. Immunol. 174 (2005) 284-293, https://doi.org/10.4049/jimmunol.174.1.284.

[25] H.S. Goodridge, E.H. Wilson, W. Harnett, C.C. Campbell, M.M. Harnett, F.Y. Liew, Modulation of macrophage cytokine production by ES-62, a secreted product of the filarial nematode Acanthocheilonema viteae, J. Immunol. 167 (2001) 940-945, https://doi.org/10.4049/jimmunol.167.2.940.

[26] J. Doonan, F.E. Lumb, M.A. Pineda, A. Tarafdar, J. Crowe, A.M. Khan, C.J. Suckling, M.M. Harnett, W. Harnett, Protection against arthritis by the parasitic worm product ES-62, and its drug-like small molecule analogues, is associated with inhibition of osteoclastogenesis, Front. Immunol. 9 (2018) 1-13, https://doi.org/10.3389/ fimmu.2018.01016.

[27] K.V.N. Rao, K. Ramaswamy, Cloning and expression of a gene encoding Sm16, an anti-inflammatory protein from Schistosoma mansoni, Mol. Biochem. Parasitol. 108 (2000) 101-108, https://doi.org/10.1016/s0166-6851(00)00209-7.

[28] K. Brännström, M.E. Sellin, P. Holmfeldt, M. Brattsand, M. Gullberg, The Schistosoma mansoni protein Sm16/SmSLP/SmSPO-1 assembles into a nine-subunit oligomer with potential to inhibit toll-like receptor signaling, Infect. Immun. 77 (2009) 1144-1154, https://doi.org/10.1128/IAI.01126-08.

[29] S. Fukumoto, M. Hiroi, P. Dirgahayu, K. Miura, S. Tademoto, H. Otsuki, Y. Ohmori, Suppression of IP-10/CXCL10 gene expression in LPS- and/or IFN- $\gamma$-stimulated macrophages by parasite-secreted products, Cell. Immunol. 276 (2012) 101-109, https://doi.org/10.1016/j.cellimm.2012.04.007.

[30] Z. Wu, L. Wang, Y. Tang, X. Sun, Parasite-derived proteins for the treatment of allergies and autoimmune diseases, Front. Microbiol. 8 (2017) 1-13, https://doi. org/10.3389/fmicb.2017.02164.

[31] K.D. Łysoniewska, K. Krawczak, K. Bocian, M. Doligalska, The effects of intestinal nematode L4 stage on mouse experimental autoimmune encephalomyelitis, Arch. Immunol. Ther. Exp. 66 (2018) 231-243, https://doi.org/10.1007/s00005-0170489-z. 